\title{
Saltation of particles in turbulent channel flow
}

\author{
Chunning Ji, ${ }^{1}$ Ante Munjiza, ${ }^{2}$ Eldad Avital, ${ }^{2}$ Dong Xu, ${ }^{1}$ and John Williams ${ }^{2}$ \\ ${ }^{1}$ State Key Laboratory of Hydraulic Engineering Simulation and Safety, Tianjin University, Weijin Road, Nankai District, \\ Tianjin 300072, People's Republic of China \\ ${ }^{2}$ School of Engineering \& Materials Science, Queen Mary, University of London, Mile End Road, London E1 4NS, United Kingdom
}

(Received 22 October 2012; revised manuscript received 15 September 2013; published 7 May 2014)

\begin{abstract}
This paper numerically investigates particle saltation in a turbulent channel flow having a rough bed consisting of two to three layers of densely packed spheres. The Shields function is 0.065 which is just above the sediment entrainment threshold to give a bed-load regime. The applied methodology is a combination of three technologies, i.e., the direct numerical simulation of turbulent flow; the combined finite-discrete element modeling of the deformation, movement, and collision of the particles; and the immersed boundary method for the fluid-solid interaction. It is shown that the presence of entrained particles significantly modifies the flow profiles of velocity, turbulent intensities, and shear stresses in the vicinity of a rough bed. The quasi-streamwise-aligned streaky structures are not observed in the near-wall region and the particles scatter on the rough bed owing to their large size. However, in the outer flow region, the turbulent coherent structures recover due to the weakening rough-bed effects and particle interferences. First- and second-order statistical features of particle translational and angular velocities, together with sediment concentration and volumetric flux density profiles, are presented. Several key parameters of the particle saltation trajectory are calculated and agree closely with published experimental data. Time histories of the hydrodynamic forces exerted upon a typical saltating particle, together with those of the particle's coordinates and velocities, are presented. A strong correlation is shown between the abruptly decreasing streamwise velocity and increasing vertical velocity at collision which indicates that the continuous saltation of large-grain-size particles is controlled by collision parameters such as particle incident angle, local bed packing arrangement, and particle density, etc.
\end{abstract}

DOI: 10.1103/PhysRevE.89.052202

PACS number(s): 92.40.Gc, 45.70.Mg, 47.27.nd, 47.11.-j

\section{INTRODUCTION}

Sediment transport is important for predicting the impact of human intervention in river and coastal systems, which can have large- and small-scale, and near- and far-field consequences. In the bed-load layer, sediment may be transported via three modes: sliding, rolling, or saltation, and the last one is considered to be the most dominant $[1,2]$. Particle saltation in turbulent channel flow has been experimentally investigated for several decades. Many statistical features, such as saltation length and height, mean particle streamwise velocity, incidence and takeoff angles at collision, dynamic friction coefficient, etc., were reported in the work of van Rijn [3], Abbott and Francis [4], Niño and García [5,6], Niño et al. [7], Lee and Hsu [8], Lee et al. [9,10], and Ancey et al. [11,12]. However, higher-order statistics of the particle translation and rotation, the statistical features of the particle-laden horizontal turbulent channel flow, and the dynamic process of the particles impacting on and rebounding from the rough bed have not been studied adequately due to the fact that they are very difficult, if not impossible, to be measured directly.

Besides experimental studies, several different theoretical models were presented by Lee and Hsu [8], Lee et al. [9], Osanloo et al. [13], and Niño and García [1]. These models are based on Newton's second law and some vital parameters, such as drag and lift coefficients, restitution and friction coefficients, incident and takeoff angles, etc., are determined experimentally and reasonable results on the first-order statistics were obtained. However, the fluctuation in particle trajectories caused by the turbulent coherent structures and the diversity of takeoff angles owing to the random packing arrangement of particles forming the rough bed were not reproduced in those results. Niño and García [14] used a random walk approach to model the turbulent fluid velocity and stated that neglecting turbulence will lead to the overestimation of the mean values of the saltation length and height. Bialik et al. [2] investigated the influence of turbulence structures on the trajectories of saltating particles in two-dimensional (2D) steady open-channel flow. The instantaneous longitudinal fluctuating velocity was generated by using a special procedure based on the assumption that the spectrum of turbulent kinetic energy is known at any distance from the bed.

With the development of high-performance computing technology and numerical schemes, sediment transport simulation in which the particles are well resolved by grids presents an effective way not only to investigate the interaction between particle motions and near-bed turbulence structures, but also to show the dynamic process of particle saltation in detail in which the hydrodynamic forces and moments, collision between particles, and pressure distributions on the particle surface are investigated.

Chan-Braun et al. [15] numerically investigated the hydrodynamic forces and torques on spherical particles fixed on a bed consisting of one layer of spheres in a square arrangement using a combination of direct numerical simulation (DNS) for the turbulent flow and the immersed boundary method (IBM) for the fluid-solid interactions. Chan-Braun et al. [16] and Kidanemariam et al. [17] further investigated the statistical features of finite-size heavy particles' transport in turbulent open-channel flow. Shao et al. [18] carried out a fully resolved DNS of particle-laden turbulent flow in a horizontal channel by using the direct-forcing fictitious domain method which is closely related with IBM. These results contributed greatly to the understanding of the underlying physical mechanisms of particle transport in turbulent channel flow. 
However, in the studies of Chan-Braun et al. [16], Kidanemariam et al. [17], and Shao et al. [18], the particles were idealized as rigid spheres and their collisions and resulting contact forces were calculated using an artificial repulsion potential. The tangential friction force (and corresponding moment) due to the frictional-sliding contact was neglected which can significantly change the rotation of the particles and thus change the hydrodynamic lift force due to the wellknown Magnus effect, especially when the collision likelihood is relatively high. Alternatively, Ji et al. [19] studied the interaction between turbulent coherent structures and particle entrainment by coupling DNS and the IBM together with the combined finite-discrete element method (FDEM) which takes into account particle deformability, frictional contact forces, and frictional and plastic loss of energy. Particle movement, hydrodynamic forces, and turbulent flow statistics at the incipience of entrainment were reported. This paper further investigates the subsequent continuous saltation after the particles have been entrained by the turbulent coherent structures. It reports on the interaction between the saltating particles and the turbulent coherent structures, the statistical features of the particle-laden flow, and the dynamic process of saltation in a turbulent channel flow.

The structure of the paper is as follows. In Sec. II we introduce the methodology. After that, in Sec. III, statistical features of particle-laden turbulent flow and particle motion, together with the profiles of the sediment concentration and the volumetric flux density, are presented. Several key parameters of particle saltation trajectory are compared with the published data. Then in Sec. IV, the dynamic process of a typical particle saltation and its physics are discussed. Finally, we present conclusions as Sec. V.

\section{METHODOLOGY}

The whole problem can be divided into three subproblems, i.e., the simulation of turbulent channel flow; the simulation of particle movement, deformation, and collision; and the simulation of the interaction between turbulent flow and particle motion. Accordingly, the methodology applied in this study is a combination of three cutting-edge numerical technologies and the corresponding computing codes.

The code used to simulate the turbulent flow is an in-house computational fluid dynamics (CFD) $\mathrm{C}$ code called CGLES [20]. It is a three-dimensional (3D) DNS-LES (large-eddy simulation) code which solves the Navier-Stokes equations using a finite volume method with second-order accuracy in both time and space. This code was applied to simulate turbulent flow over a rough bed in our previous work [21,22] and has a proven high fidelity and parallelizing efficiency.

The immersed boundary method, first introduced by Peskin [23] in the simulation of blood flow around the flexible leaflet of human heart, was incorporated into CGLES to model the interaction between the flow and moving particles. In the IBM framework, the flow governing equations are discretized on a fixed Cartesian grid which generally does not conform to the geometry of moving solids. As a result, the boundary conditions on the fluid-solid interface which manifest the interaction between fluid and solid cannot be imposed straightforwardly. Instead, the solid surface is represented by a set of immersed boundary points (IBPs) on which an extra singular body force is imposed. This force is then added into the momentum equation of fluid motion to take such fluid-solid interaction into account using interpolation-distribution functions.

The conservative form of the second-order AdamsBashforth temporal-discretized governing equations of incompressible fluid flow using the IBM are

$$
\begin{aligned}
\mathbf{u}^{n+1}= & \mathbf{u}^{n}+\delta t\left(\frac{3}{2} \mathbf{h}^{n}-\frac{1}{2} \mathbf{h}^{n-1}-\frac{3}{2} \nabla p^{n}+\frac{1}{2} \nabla p^{n-1}+\mathbf{g}\right) \\
& +\mathbf{f}^{n+\frac{1}{2}} \delta t
\end{aligned}
$$

and

$$
\nabla \cdot \mathbf{u}^{n+1}=0
$$

where $\mathbf{u}$ is the fluid velocity, $p$ is the pressure, $\mathbf{g}$ is the gravitational acceleration, $\mathbf{h}=\nabla \cdot\left[-\mathbf{u u}+v\left(\nabla \mathbf{u}+\nabla \mathbf{u}^{t}\right)\right]$ is comprised of the convective and diffusive terms, and the superscript $n$ is the time step. The extra body force $\mathbf{f}$ on the Cartesian grids is defined as

$$
\begin{aligned}
\mathbf{f}^{n+\frac{1}{2}}= & \frac{1}{\delta t} D\left\{\mathbf{V}^{n+1}-I\left[\mathbf{u}^{n}+\delta t\left(\frac{3}{2} \mathbf{h}^{n}-\frac{1}{2} \mathbf{h}^{n-1}-\frac{3}{2} \nabla p^{n}\right.\right.\right. \\
& \left.\left.\left.+\frac{1}{2} \nabla p^{n-1}+\mathbf{g}\right)\right]\right\},
\end{aligned}
$$

where $I$ and $D$ represent the interpolation and distribution functions, respectively, and $\mathbf{V}$ is the velocity of the IBPs which is determined by the motion of the solids. The spatial operators are evaluated by central finite differences on a staggered grid and the temporal and spatial accuracy of this scheme is second order.

The main advantage of the IBM is associated with its inherent simplicity in treating flows which have solids with moving boundaries, due to the fact that the IBPs are not connected with the Cartesian background grid. To improve the accuracy of the IBM, an iterative direct-forcing IBM which was introduced in our previous work [24] is applied in this study.

To simulate the movement and collision of particles, the FDEM developed by Munjiza et al. [25] was applied. In the context of the FDEM, each particle of general shape is represented as a single discrete element which is then discretized into finite elements for deformability, fracture, and fragmentation. The movement of particles is simply governed by Newton's second law. The penalty function method is employed to calculate the normal contact force $\mathbf{F}_{n}$ when two particles are in contact and assumes that the two particles penetrate each other. Because the discrete elements are discretized into finite elements, each contacting pair of discrete elements is in fact represented by a set of contacting finite elements. The elemental contact force is then directly related to the overlapping area of the finite elements in contact. In this study, a distributed contact force algorithm introduced by Munjiza and Andrews [26] — which is relatively easy to implement and preserves energy balance-is applied to generate a realistic distribution of contact forces over finite contact areas. The calculated elemental contact force is then distributed around the nodes surrounding the contact in order to preserve the system from artificial stress concentration. 
As suggested by Munjiza and Andrews [26], the normal contact force is given by

$$
\mathbf{F}_{n}=\sum_{i=1}^{n} \sum_{j=1}^{m} \int_{\Gamma_{\beta_{c i} \cap \beta_{t j}}} \mathbf{n}_{\Gamma_{\beta_{c} i \beta_{i j}}}\left(\varphi_{c i}-\varphi_{t j}\right) d \Gamma,
$$

where $\beta_{c i}$ and $\beta_{t j}$ are the $i$ th and $j$ th finite elements of the contactor and target discrete elements, respectively, while $n$ and $m$ are the total number of finite elements into which the contactor and target discrete elements are discretized. $\mathbf{n}$ is the outward unit normal to the boundary $\Gamma$ of the overlapping volume $\beta_{c i} \cap \beta_{t j} . \varphi_{c}$ and $\varphi_{t}$ are potential functions for the contactor and target discrete elements, respectively.

In this study, the simplest finite element in $3 \mathrm{D}-\mathrm{a}$ linear four-node tetrahedron-has been adopted. The potential function at an arbitrary point $P$ inside a tetrahedral finite element is given by

$$
\varphi(P)=k_{p} \min \left\{4 V_{1} / V, 4 V_{2} / V, 4 V_{3} / V, 4 V_{4} / V\right\},
$$

where $k_{p}$ is the penalty parameter, $V$ is the volume of the tetrahedral finite element, and $V_{i}(i=1,2,3,4)$ are the volumes of the corresponding subtetrahedrons consisting of the point $P$ and the faces of the tetrahedral finite element. The potential $\varphi$ equals 1 at the tetrahedron center and 0 at and beyond the tetrahedron surfaces.

Xiang et al. [27] further developed the FDEM by taking into account the sliding friction force by implementing the well-known Coulomb-type friction described as follows:

$$
\mathbf{F}_{t}=-k_{t} \mathbf{D}_{t}-\eta_{d} \mathbf{V}_{t},
$$

in which $\mathbf{F}_{t}$ is the tangential elastic contact force, $k_{t}$ the tangential spring stiffness constant, $\eta_{d}$ the viscous damping coefficient, and $\mathbf{D}_{t}$ and $\mathbf{V}_{t}$ are the tangential relative displacement and velocity between particles, respectively. If $\mathbf{F}_{t}$ is bigger than the friction force obeying the Coulomb-type friction law, i.e., $\left|\mathbf{F}_{t}\right|>\mu\left|\mathbf{F}_{n}\right|$, the particles slide over one another and the tangential force is then calculated using the total normal elastic contact force $\mathbf{F}_{n}$ :

$$
\mathbf{F}_{t}=-\mu\left|\mathbf{F}_{n}\right|\left(\mathbf{V}_{t} /\left|\mathbf{V}_{t}\right|\right),
$$

where $\mu$ is the coefficient of sliding friction.

The lubrication force generated in the microscale gap of colliding particles in viscous fluids prevents particles from touching, and thus proper account of this lubrication effect is essential to correctly calculate the particle viscous momentum losses during collisions for smooth particles at low and intermediate Stokes numbers $\mathrm{St}=\left(\rho_{s} / 9 \rho_{f}\right) \operatorname{Re}(\mathrm{Re}$ is the particle Reynolds number based on the incident particle velocity just before collision and the particle diameter). However, fully resolving this interstitial film requires the grid spacing to be extremely small; even body-fitted approaches will be computationally prohibitive for 3D simulations with a large number of particles. Instead, an analytical lubrication model is usually used to take into account this lubrication effect by imposing a singular lubrication force on the particles. It is well known that below the Stokes number of 10 the lubrication effect is significant and no rebound occurs after collision in viscous fluids. However, as stated in Ardekani and Rangel [28], for collisions characterized with a Stokes number larger than 500, the restitution coefficient $e$ asymptotes to that for a dry collision $e_{\text {dry }}$ which indicates that the lubrication force can be neglected. In our study, the Stokes number is approximately 146 when a mean incident velocity of colliding particles of $5 u_{\tau}$ is used (shown later), and thus the contribution to the momentum loss from the lubrication effect should not introduce significant error. Other reasons for not considering the lubrication force can be found in our previous work [19] and, for the sake of computational efficiency and algorithm simplicity, it is omitted.

The FDEM code therefore includes algorithmic procedures for deformability of individual discrete elements, fracture and fragmentation algorithms (switched off for this study), contact detection between moving solids, frictional-sliding interaction between solids, and a whole set of application specific algorithms. Readers should refer to Munjiza [29] and Munjiza et al. [30] for further details on the FDEM.

\section{STATISTICS OF TURBULENT FLOW AND PARTICLE TRANSPORTATION}

\section{A. Problem description}

In this study, we considered sediment transport in a fully developed turbulent open-channel flow having a rough bed consisting of two to three layers of densely packed spheres (see Fig. 1). The total number of spheres $N_{p}$ was 6355 and the rough bed was water worked, meaning that the most exposed ones were removed. This sphere arrangement [31] was provided by researchers in TU Delft. Note that the number of particles is much smaller than that for numerical sediment transport simulations in which the particles are represented by Lagrangian volumeless points, due to the fact that in our case the particles are fully grid resolved (grid spacing is $1 / 16$ of the particle diameter in all three directions) to guarantee a correct modeling of the turbulence-particle interaction. This makes the simulation with an even larger number of particles computationally prohibitive. On the other hand, the number of the particles in our simulation is sufficiently large for the investigation on the interaction between the entrained particles and the turbulent coherent structures. Similarly, Chan-Braun et al. [16] used 9216 particles, Kidanemariam et al. [17] used 518 particles, and Shao et al. [18] 2160 particles in

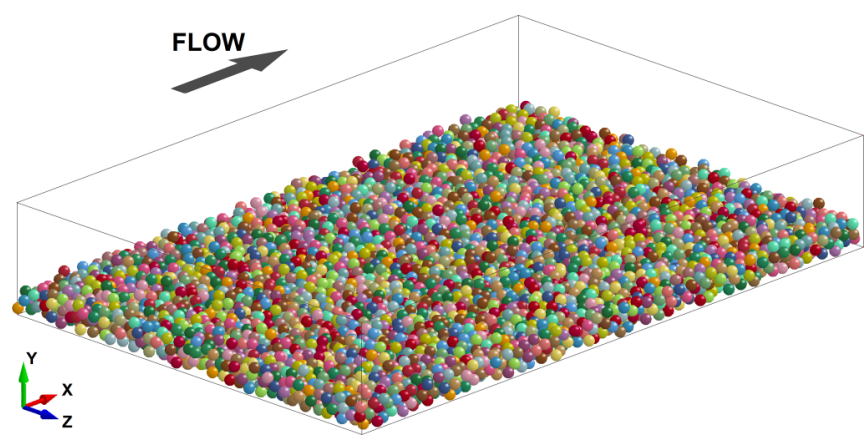

FIG. 1. (Color online) Channel geometry. The size of the computational box is $6 d \times d \times 4 d$ in $x, y$, and $z$ directions, where $d$ is the overall channel depth. The water-worked rough bed consists of two to three layers of densely packed spheres with a diameter of $D=0.1 d$ and the height of the roughness elements from the channel bed to the highest crests of the spheres is $k=0.3 d$. 


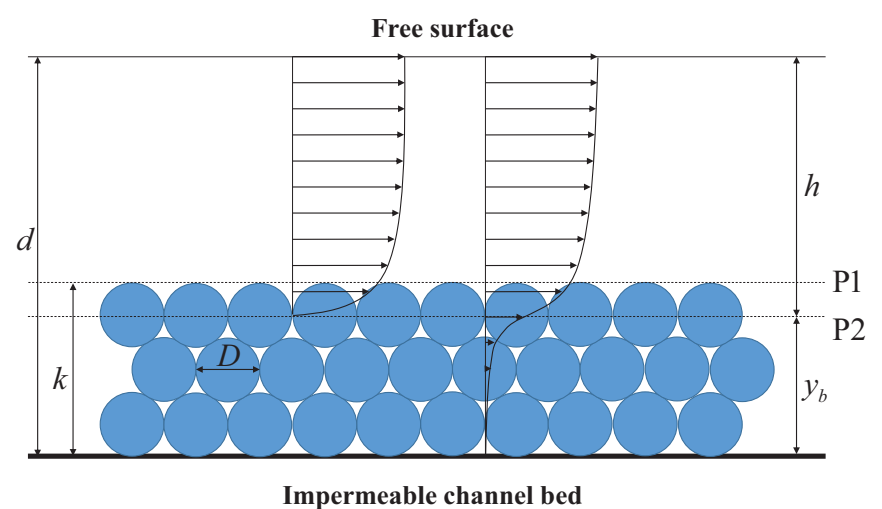

FIG. 2. (Color online) Illustration of turbulent flow over a rough bed consisting of densely packed spheres. $d$ is the overall channel depth, $k$ the roughness height, $D$ the sphere's diameter, $h$ is the effective channel depth, and $y_{b}$ is the effective bed location. P1 represents the highest crests of the roughness elements and P2 represents the effective channel bed. The right profile denotes a possible mean velocity profile of turbulent flow over a permeable rough bed, while the left one denotes that of turbulent flow over an imaginary smooth bed at P2.

their numerical simulation of particle-laden turbulent flow in a horizontal open channel.

The computational domain is a 3D box with a size of $6 d \times d \times 4 d\left(L_{x} \times L_{y} \times L_{z}\right)$, where $d$ is the overall channel depth. The rough bed consists of two to three layers of densely packed spheres with a diameter of $D=0.1 d$ and the height of the roughness elements from the channel bed to the highest crests of the spheres is $k=0.3 d$; see Fig. 2. The turbulent channel flow is driven by a horizontal body force which is balanced by the shear stresses on the rough bed. It is well known that, for fully developed wall-bounded turbulent flow, the normalized mean streamwise velocity profile verifies the logarithmic law of the wall $u^{+}=(1 / \kappa) \ln y^{+}+C^{+}$if the velocity $u$ is made dimensionless by the friction velocity $u_{\tau}=\sqrt{\tau_{w} / \rho_{f}}$ and the wall distance $y$ is made dimensionless by the viscous length $l=v / u_{\tau}$-commonly used velocity and length scales to describe turbulent flow in a boundary layer. Here, $\kappa \approx 0.41$ is the von Kármán constant, $v$ is the fluid kinematic viscosity, $C^{+}$is an empirical constant and approximately equal to 5.0 for a smooth-bed turbulent flow, $\tau_{w}$ is the bed shear stress, and the superscript "+" indicates quantities in wall units, i.e., velocity and length are normalized by $u_{\tau}$ and $l$, respectively. Note that, for the turbulent flows over a smooth bed, there is a clear definition of where the bed is and what the channel depth is. However, for turbulent flows over a rough bed, the definitions of channel bed and depth are ambiguous. A commonly used approach is to fit the mean velocity profile of the rough-bed flow to the one for smooth-bed flow, i.e., finding proper values of the effective bed location $y_{b}$ and the equivalent roughness height $k_{s}$ to make the mean velocity profile comply with the logarithmic law of the wall using a curve-fitting approach. Applying this procedure to our numerical results - the mean velocity profile of the single-phase flow shown in Fig. 3-we have $y_{b}=$ $0.252 d$ and $k_{s}=0.242 d$, and thus the effective channel depth is calculated as $h=d-y_{b}=0.748 d$. Note that the

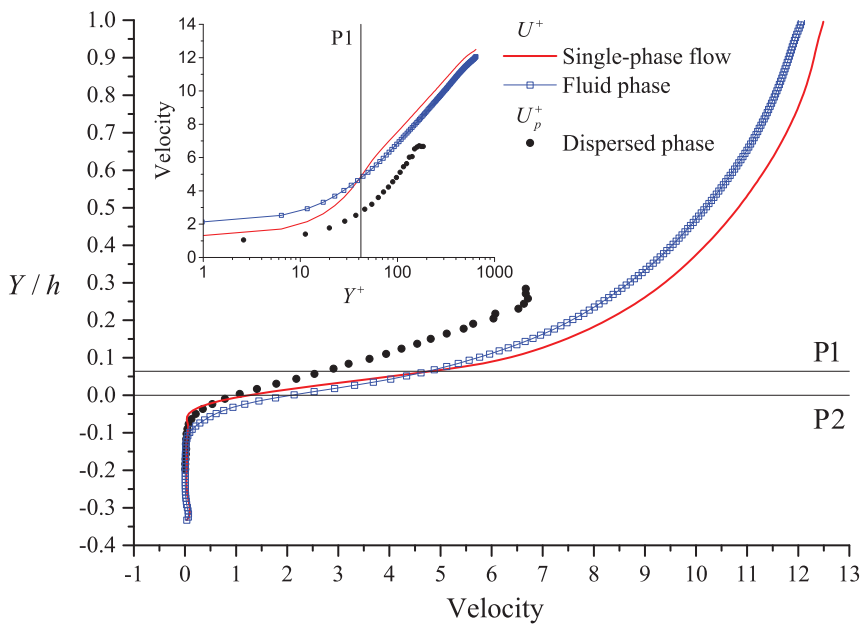

FIG. 3. (Color online) Comparison of the mean streamwise velocity profiles. The inset shows a semilog plot of the velocity profiles. $U^{+}$is the plane and time-averaged streamwise velocity of turbulent flow and $U_{p}^{+}$is the binned and time-averaged streamwise velocity of the entrained particles. P1 and P2 are two wall-parallel planes and their meanings are shown in Fig. 2.

$\tau_{w}$ adopted in this study is the shear stress at $y=y_{b}$. Two wall-parallel planes are defined as $\mathrm{P} 1$ - a plane at the highest crests of the roughness elements $(y=k)$, and P2 - a plane at the effective bed location $\left(y=y_{b}\right)$; see Fig. 2 .

Other flow parameters are as follows. The Reynolds number of the turbulent channel flow (the effective channel depth in wall units) is $\operatorname{Re}_{h}^{+}=u_{\tau} h / v=647$, the particle Reynolds number (the particle size in wall units) is $\operatorname{Re}_{D}^{+}=$ $u_{\tau} D / v=86.5$, and the roughness Reynolds number (the equivalent roughness height in wall units) is $k_{s}^{+}=u_{\tau} k_{s} / \nu=$ 209 indicating a completely rough flow regime. The density ratio is $s_{s}=\rho_{s} / \rho_{f}=2.65$ and the Shields function $\Theta=\tau_{w} /$ $\left[\left(\rho_{s}-\rho_{f}\right) g_{y} D\right]=0.065$ is just above the threshold for sediment entrainment $\left(\Theta_{c}=0.06\right)$ which indicates a bed-load regime. Here, $\rho_{s}$ is the particle density and $g_{y}$ is the vertical component of the gravitational acceleration.

The computational domain is discretized on a uniform Cartesian grid with $960 \times 160 \times 640\left(N_{x} \times N_{y} \times N_{z}\right)$ grid nodes which yields a grid spacing in wall units of $\Delta x^{+}=$ $\Delta y^{+}=\Delta z^{+} \approx 5.4$. No-slip boundary conditions were used on both the bed and sphere surfaces and the top boundary was set as a free-slip hard lid. Periodicity was imposed in both the streamwise and spanwise directions.

The following parameters were applied in the FDEM for the modeling of particle collisions. The Young's modulus is $E=10 \mathrm{MPa}$, Poisson ratio $\gamma=0.3$, particle density $\rho_{s}=$ $2650 \mathrm{~kg} / \mathrm{m}^{3}$, the coefficient of sliding friction $\mu=0.4$, and the particle diameter is $D=0.001 \mathrm{~m}$. The penalty parameter is calculated as $k_{p}=10 \lambda$ and the tangential spring stiffness constant is determined as $k_{t}=k_{p} / 10$, where $\lambda=$ $E \gamma /[(1+\gamma)(1-2 \gamma)]$ is the Lamé's first parameter. The viscous contact damping is determined as $\eta_{d}=2 h_{e} \sqrt{E \rho_{s}}$, where $h_{e}$ is the smallest size of the finite elements. Note that the value of the Young's modulus used here is rather lower than the realistic one; e.g., $E$ for sand is typically $10 \sim 70 \mathrm{GPa}$. However, a large value of $E$ will lead to an extremely small 
time step due to contact detection and computational stability, which is a significant limitation to large-scale simulations. It is shown in Simeonov and Calantoni [32] that the dynamics of granular flows is rather insensitive to the value of $E$ and their results show that the difference is less than $4 \%$ in the coefficient of restitution for material stiffness, estimated as $E D / 2$, in the range of $10^{3}-10^{6} \mathrm{~N} / \mathrm{m}$.

The computations have been run on the UK's national supercomputing facility: HECToR. This simulation was carried out on 150 processors (AMD Opteron $2.3 \mathrm{GHz}$ ) for a wall-clock time of 2 months, which is about $200 \mathrm{~K} \mathrm{CPU}$ hours. It should be mentioned here that the dimensionless grid resolution in all three directions is approximately 5.4 which means the DNS of turbulent flow is under-resolved near the wall and near the surface of the particles. We acknowledge the limitation of our simulation. A simulation with finer grid resolution will be considered in future.

In order to keep the computational requirements to a minimum, we first carried out a LES at one half of the DNS grid resolution with fixed spheres. The LES was run for around $30 T$ (where $T=d / u_{\tau}$ is the large-eddy turnover time) to obtain a fully developed turbulence flow. This fully developed turbulent flow field was then interpolated onto the DNS grid to yield the initial conditions for the DNS. Time integration was performed on the DNS grid for $20 T$ to make the effect of the initial conditions vanish and for the velocity field to reach a fully developed state. The mean velocity profiles and turbulent quantities were calculated over a further $10 T$. The choice of the simulation time for LES and DNS is long enough to guarantee a fully developed turbulent flow, which can be confirmed by the linear profile of the total shear stress shown in Fig. 7. Once the turbulent flow was statistically stable, the spheres were set free and their motions together with the surrounding flow fields were recorded for a further $12 T$. First- and second-order statistics of the particle-laden turbulent flow and the particle transport were calculated over the last $6 T$.

To facilitate the following analysis and discussion, the following terminology is adopted: Single-phase flow indicates the turbulent channel flow over the fixed rough bed, and twophase flow denotes the turbulent channel flow with sediment transport. The fluid phase of the two-phase flow represents the flow in the whole computational domain excluding the volume occupied by spheres regardless of their state of motion while the dispersed phase of the two-phase flow is the moving spheres. Furthermore, in order to compare the two-phase flow results with the single-phase ones from our previous work [19], a vertical coordinate $Y=y-y_{b}$ is adopted.

\section{B. Single-phase turbulent flow verification}

The statistical features of the single-phase flow were verified with available experimental data and numerical results. Good agreement on velocity profiles, turbulence intensities, and shear stresses have been reported in our previous work [19]. A grid convergence study was also carried out on two meshes with increasing refinement. The grid resolution increased from the coarser mesh to the finer mesh so that the number of grids doubled in each direction, and it was found that the flow parameters for the two meshes were virtually identical which verified that the numerical results were grid independent. Other verifications including the distance from the effective bed location to the top of the roughness elements have also been reported in Ref. [19].

\section{Statistical features of the fluid phase and the turbulent coherent structures in the two-phase turbulent flow}

Figure 3 shows the plane (binned) and time-averaged streamwise velocity of the fluid (dispersed) phase of the two-phase flow, together with that of the single-phase flow for comparison. Here, the plane averaging of Eulerian quantities on the DNS grids was performed over the fluid-occupied domain in the wall-parallel planes and the binned averaging of the Lagrangian quantities related to particles was performed over the particles with their center in the wall-parallel bins with a thickness of $D / 10$. A total of 12000 flow field snapshots during a time span of $6 T$ was used for the time averaging. As seen in Fig. 3, the velocity profile of the fluid phase is retarded above $\mathrm{P} 1$, comparing with that of the single-phase flow, due to the presence of the entrained particles. Below this position, the flow is accelerated because the moving particles cause less momentum exchange with the fluid phase. The mean streamwise velocity of the dispersed phase is always smaller than that of the fluid phase and shows a quasilinear profile although there are small fluctuations in the top region of the bed-load layer $(y / h>0.2)$ due to the sampling of an insufficient number of particles visiting there. The height of the bed-load layer, calculated as the distance from P2 to the highest position that the moving particles' center can reach, is approximately $0.29 h(2.1 \mathrm{D})$. The mean velocity profile of the fluid phase verifies the logarithmic law of the wall above $Y / h=0.29$, as shown in the inset of Fig. 3. However, the transition from a concave curve to a convex one as shown in the mean velocity profile of the single-phase flow is absent from the mean velocity profile of the fluid phase.

Figure 4 shows the streamwise velocity lag between the fluid and dispersed phases. It peaks roughly at P1 and then decreases with height although there is an upward tip observed at the top region of the bed-load layer mainly due to insufficient sampling. This velocity lag is well documented in the experiments of Kaftori et al. [33], Taniere et al. [34], Kiger and Pan [35], Righetti and Romano [36], and Muste et al. [37], as well as the numerical simulations of Chan-Braun et al. [16], Kidanemariam et al. [17], and Shao et al. [18]. Chan-Braun et al. [16] and Kidanemariam et al. [17] attribute this feature to the fact that particles preferentially accumulate in the streaks of low-speed fluid regions [referred to as the streaky structures hereafter; see Fig. 6(a)] near the bottom wall and that these low-momentum particles are then ejected into the outer flow region by counter-rotating vortices-refer to Fig. 10 of Kidanemariam et al. [17]. However, it is not the case for the present study that the streaky structures disappear and the particles scatter in the near-wall regionsee Fig. 5. The reasons for this discrepancy are discussed below.

The coherent structures, i.e., the streaky patterns of lowspeed and high-speed flow regions near the wall, are the characteristic features of turbulent flow over a smooth bed. These streaky patterns can be clearly observed in Fig. 10(b) of Kidanemariam et al. [17], in which the contours of the 


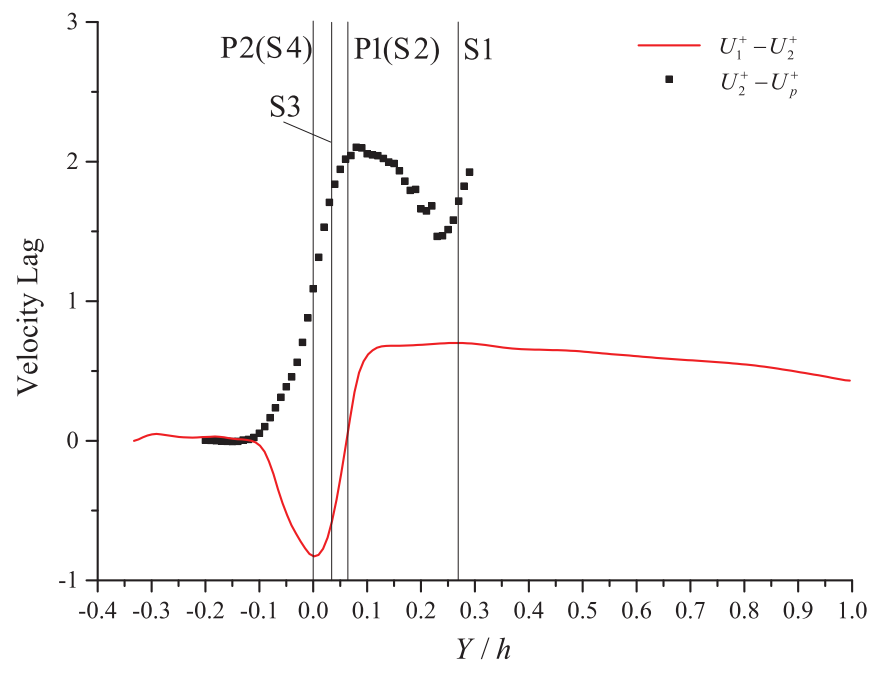

FIG. 4. (Color online) Velocity lag profiles. $U_{1}^{+}$and $U_{2}^{+}$are the plane and time-averaged streamwise fluid velocities of the singlephase and two-phase flow, respectively. $U_{p}^{+}$is the binned and timeaveraged streamwise particle velocity of the dispersed phase. P1 and $\mathrm{P} 2$ are two wall-parallel planes and S1-S4 are four wall-parallel slices. P1 and P2 coincide with S2 and S4, respectively.

instantaneous normalized streamwise fluid velocity fluctuations in a wall-parallel plane located at $y^{+}=5$ (in wall units) are plotted. For flow over a rough bed, however, the streaky structures are gradually undermined when the normalized

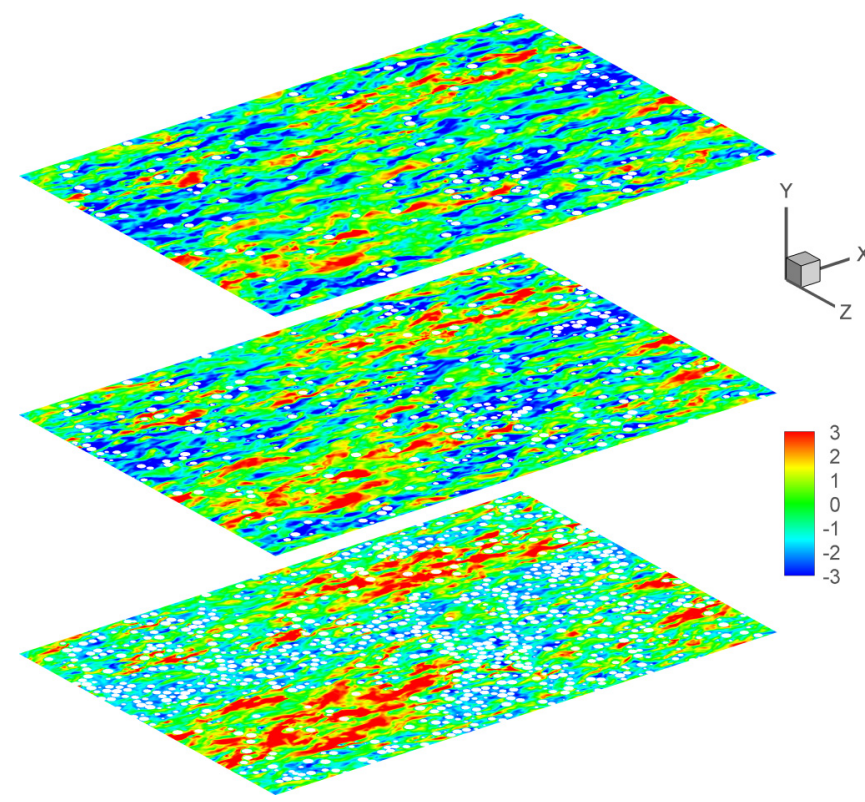

FIG. 5. (Color online) Visualization of instantaneous normalized streamwise fluid velocity fluctuations $u^{\prime} / u_{\tau}$ in three wall-parallel slices located at $Y^{+}=0.0$ (S4), 21.6 (S3), and 43.3 (S2) (from bottom to top), respectively. The positions of the three slices are shown in Fig. 4 and the intersection of the particles and the slices are represented by the small white areas which are preferentially distributed in the low-speed fluid region at S4 but scatter at S2 and S3. The well-known quasi-streamwise-aligned streaky structures cease to exist in the near-wall region. roughness height is larger than 5 wall units (i.e., the roughness elements protrude from the viscous sublayer whose thickness is 5 wall units) and are completely destroyed when the normalized roughness height is larger than 50-100 wall units, as stated in Jiménez [38]. In our single-phase flow case, the particle Reynolds number is 86.5 which means the roughness elements significantly protrude from the viscous sublayer and thus the streaky structures are not observed in the near-wall region.

For particle-laden flows with relatively small particles, the particles preferentially accumulate in the low-speed region due to counter-rotating vortices, as shown in Fig. 10(a) of Kidanemariam et al. [17]. In their simulation, the particle Reynolds number, $\mathrm{Re}_{D}^{+}$, (normalized particle size) was 7.2 and the solid volume fraction, $\Phi_{s}, 0.05 \%$; thus the influences of particle motion on the streaky structures was neglectable. However, in our particle-laden flow case, these two values are much higher, 86.5 and $13.9 \%$, respectively_see Table I for the flow configuration and particle parameters of different studies - and the existence of the entrained particles significantly modifies the turbulent flow. This can be seen in Fig. 5 in which particle locations (white areas) are plotted on the wall-parallel slices S2 (at $Y^{+}=43.3$ which coincides with $\mathrm{P} 1$ ) and S3 (at $Y^{+}=21.6$ ), together with the contours of the instantaneous streamwise fluid velocity fluctuations in wall units. On S2 and S3, the streaky structures disappear (note that the small low-speed fluid regions behind the particles are actually the wakes instead of the low-speed streaks generated by counter-rotating vortices) and the particles are not concentrated in the low-velocity regions, as in Kidanemariam et al. [17]. Shao et al. [18] state that the large-scale streamwise vortices (i.e., the streaky structures) are suppressed by the particles due to the fact that the particles entrained serve as a dissipative source. In the experimental study of Hetsroni and Rozenblit [39], the authors report that particles having a diameter of more than 30 wall units do not accumulate in the low-speed fluid regions which, again, clearly supports our findings. Although the particle distribution on $\mathrm{S} 4$ (at $Y^{+}=0.0$ which coincides with P2), shows some sort of preference as particles are accumulated in the low-speed fluid regions, this, however, is not related to turbulent coherent structures but to the increased local drag on the flow-accumulated particles have larger resistances due to the interlocking effects between themselves and the roughness elements - thereby reducing the local fluid velocity.

We further investigated instantaneous streamwise velocity fluctuations on the wall-parallel slice $\mathrm{S} 1\left(Y^{+}=174\right.$, two particle diameters up from $\mathrm{P} 2$ ) in the outer flow region and found that beyond the vertical extent of entrained particles, turbulent coherent streaks recover; see Fig. 6(a). The streaky structures are obviously elongated-a result that is in agreement with the findings of Singh et al. [21]. The dimensions of the low-speed fluid regions (streaky structures) are tens of particle diameters in length (restricted by the computational box size in the $x$ direction) and several particle diameters in width, both of which are much larger than those in Fig. 5 (near wake) that are approximately several particle diameters in length and one particle diameter in width. Figure 6(b) shows the instantaneous streamwise velocity in a cross-section plane in which the streaky structures can be clearly observed above $y / d=0.4$ 

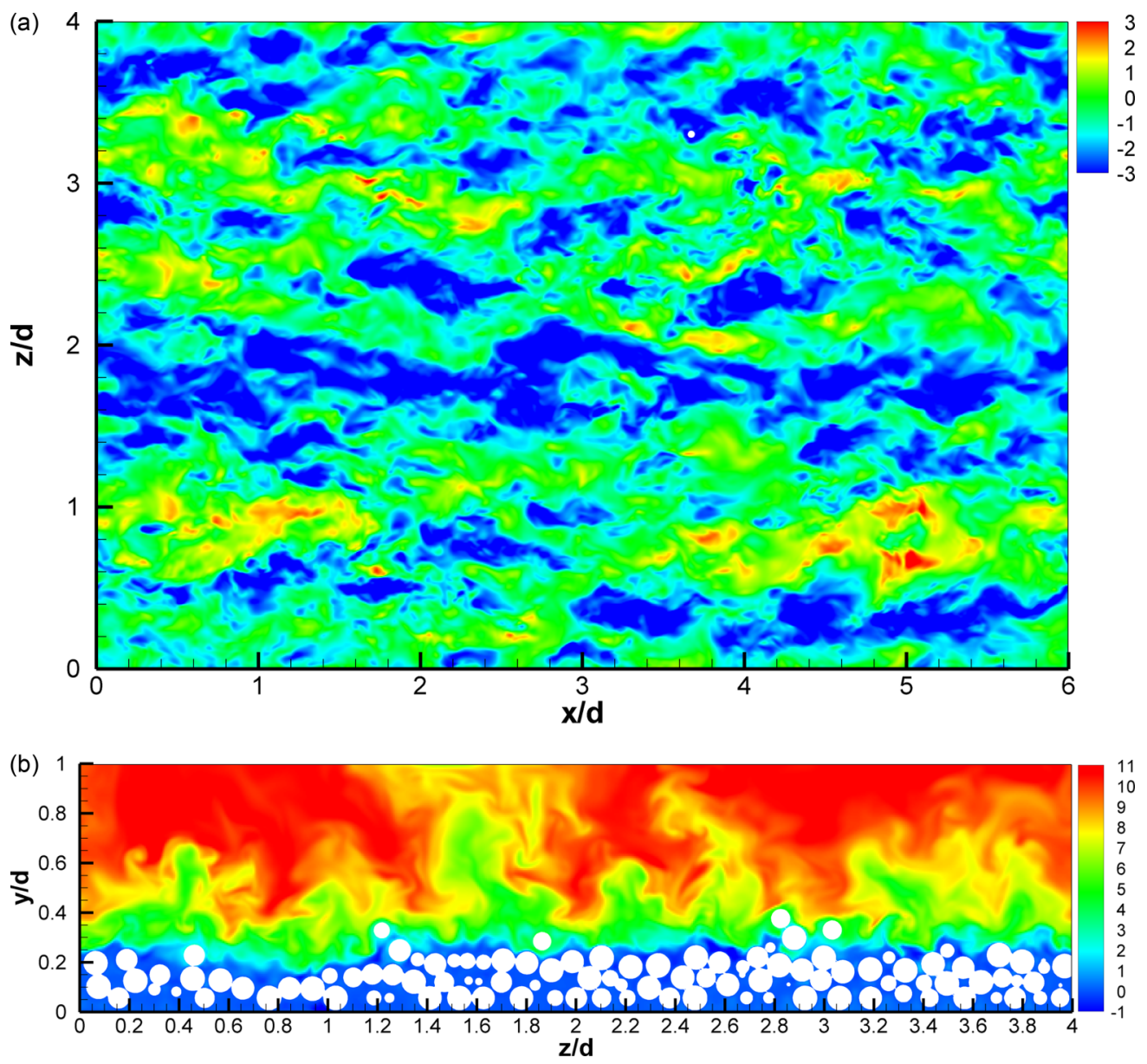

FIG. 6. (Color online) Visualization of the streaky structures in a particle-laden flow. (a) Instantaneous normalized streamwise fluid velocity fluctuations $u^{\prime} / u_{\tau}$ in a wall-parallel slice $\mathrm{S} 1$ located at $Y^{+}=174$ where the entrained particles are hard to reach and the elongated streaky structures are observed. (b) Instantaneous streamwise fluid velocity in a cross-section plane. White areas represent the intersection of the particles with the planes.

$\left(Y^{+}=128\right)$. It can therefore be concluded (i) that very rough beds (not only caused by the large-grain-size particles but also by random packing of the roughness elements) and entrained large particles have a tendency to destroy coherent streaky structures in the near-wall region, and (ii) that beyond where the rough-bed effect is weak and entrained particles rarely visit, turbulent coherent structures begin to recover.

TABLE I. The flow configuration and particle parameters of different numerical simulations. $L_{i}$ and $N_{i}$ are the computational box length and number of grid points in the $i$ th coordinate direction, respectively. $N_{p}$ is the number of spherical particles, $h$ the effective channel depth, $D$ the particle diameter, and $\Delta x$ (equal to $\Delta y$ and $\Delta z$ in all these studies) and $\Delta x^{+}$are the grid spacing and the normalized grid spacing in $x$ direction, respectively. $\operatorname{Re}_{h}^{+}$is the Reynolds number based on $h$ and the friction velocity $u_{\tau}$ while $\operatorname{Re}_{D}^{+}$is the Reynolds number based on $D$ and $u_{\tau} . s_{s}$ is the density ratio between solid and fluid, $\Theta$ is the Shields function, and $\Phi_{s}$ is the solid volume fraction.

\begin{tabular}{lcccc}
\hline \hline Variables & Chan-Braun et al. [16] & Kidanemariam et al. [17] & Shao et al. [18] & Present study \\
\hline$L_{x} \times L_{y} \times L_{z}$ & $12 d \times d \times 3 d$ & $12 d \times d \times 3 d$ & $4 d \times d \times 2 d$ & $6 d \times d \times 4 d$ \\
$N_{x} \times N_{y} \times N_{z}$ & $1536 \times 129 \times 384$ & $3072 \times 257 \times 768$ & $256 \times 64 \times 128$ & $960 \times 160 \times 640$ \\
$N_{p}$ & 9216 & 518 & $30-2160$ & 6355 \\
$h / D$ & 21 & 25.6 & 10 and 20 & 7.48 \\
$D / \Delta x$ & 6 & 10 & 207.5 & 16 \\
$\operatorname{Re}_{h}^{+}$ & 208 & 184 & 10.4 and 20.8 & 647 \\
$\operatorname{Re}_{D}^{+}$ & 10 & 7.21 & 3.24 & 86.5 \\
$\Delta x^{+}$ & 1.7 & 0.72 & 1.5 & 5.4 \\
$s_{s}$ & 1.7 & 1.7 & 0.111 and 0.222 \\
$\Theta$ & 0.21 & 0.19 & $0.79 \%-7.08 \%$ \\
$\Phi_{s}$ & $1.4 \%$ & $0.05 \%$ & 0.065 \\
\hline \hline
\end{tabular}




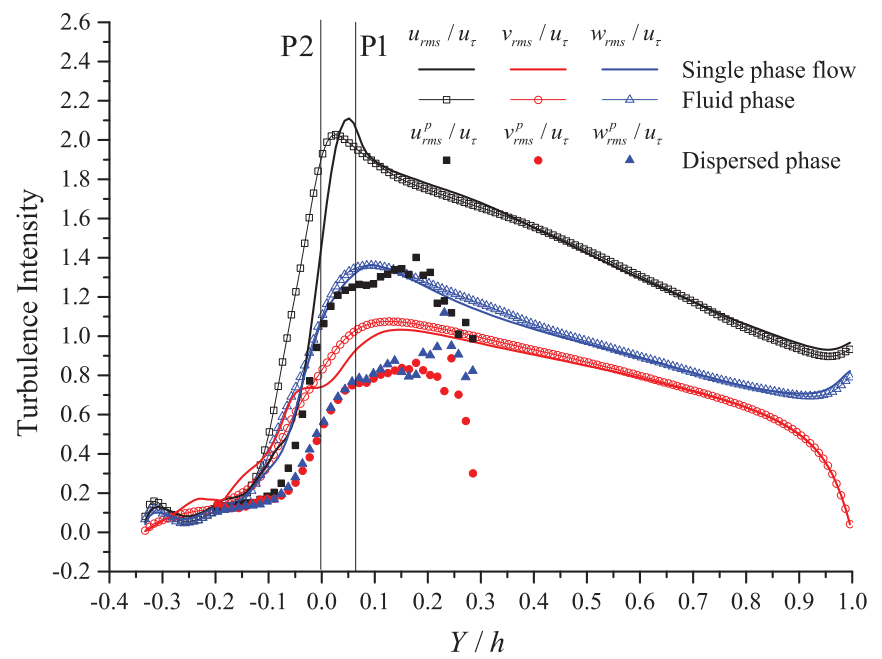

FIG. 7. (Color online) Comparison of turbulence quantities between the single-phase and two-phase flow cases. The subscript " $r m s$ " indicates the root-mean-square value of a quantity and the superscript " $p$ " denotes a variable of the dispersed phase. P1 and P2 are two wall-parallel planes and their positions are shown in Fig. 2.

The streamwise fluid velocity lag between the single- and the two-phase flows is also presented in Fig. 4 and shows a negative peak at $\mathrm{P} 2$ which then increases sharply with height until it reaches a horizontal step at $Y / h \approx 0.1$. The maximum is attained at a position coinciding with the highest position that entrained particles can reach. After that, it decreases slowly with height which means that the streamwise velocity profile of the fluid phase recovers slightly from the retardation caused by the presence of the dispersed phase.

Figure 7 shows the turbulence quantities, i.e., the normalized root-mean-square (rms) velocities of the turbulent flow and the particles, for both the single- and two-phase flow cases. The modifications to the turbulence quantities due to the presence of the dispersed phase are clearly seen, especially near and below P1. Specifically, the near-wall peak of the streamwise component $u_{r m s} / u_{\tau}$ of the fluid phase is smoothed and shifted downward and also decreases slightly in magnitude compared with that of the single-phase flow. This is in agreement with the experimental results of Kiger and Pan [35] and the numerical results of Chan-Braun et al. [16] and Shao et al. [18]. However, the fluid velocity fluctuations deviate only marginally from the single-phase counterpart in the numerical results of Kidanemariam et al. [17] which is attributed to their small solid volume fraction of $0.05 \%$. It should be noted that the near-wall peak values of $u_{r m s} / u_{\tau}$ for the single- and two-phase flow cases of the present study are approximately 2.1 and 2.0, respectively, which are much lower than the value of 2.7 for the particle-free and particle-laden cases in Kidanemariam et al. [17], owing to the streaky structures being destroyed by the large particle size and solid volume fraction in the present study. This lower-peak trend is also shown in the results of Shao et al. [18] and becomes more pronounced with increasing particle size and solid volumetric fraction. The spanwise velocity fluctuations of the two-phase flow are only marginally different from the single-phase case. However, the vertical velocity fluctuations show a perceivable increase which could be the result of high-speed particles in the outer region landing and colliding on the rough bed and introducing small-scale vortices in the near-wall region. Below $\mathrm{P} 2$, the turbulence intensities of the single-phase flow show small fluctuations, while the ones of the two-phase flow are smoother.

In contrast to their counterparts of the fluid phase, the turbulence intensities of the dispersed phase in the vertical and spanwise directions are almost identical in the near-wall region where the greater occurrence of collisions leads to a more isotropic distribution of sphere fluctuation energy in the vertical and spanwise directions and is in agreement with the findings of Chan-Braun et al. [16] and Kidanemariam et al. [17]. The streamwise component is generally larger than that of the other two and above $Y / h=0.2$, large fluctuations are observed on all three components due to the insufficient number of samples there. All three turbulence intensities of the dispersed phase are consistently smaller than their fluid phase counterparts and is a result that does not agree with the findings of Chan-Braun et al. [16] who found that the vertical velocity fluctuations of the dispersed phase are larger than their fluid phase counterparts in the near-wall region and the findings of Kidanemariam et al. [17] who found that both vertical and span-wise velocity fluctuations of the dispersed phase are larger than their fluid phase counterparts. This discrepancy could be due to the lower solid-fluid density ratio, i.e., $s_{s}=\rho_{s} / \rho_{f}=1.7$, and the smaller grain size in their study - lighter and smaller particles are more easily accelerated by the surrounding flow, and are thus more turbulent.

Figure 8 compares the Reynolds shear stress and total shear stress for the single- and two-phase flow cases. The total shear stress of the single-phase flow shows a linear profile above the effective bed location which indicates that the turbulent flow is fully developed. The two-phase flow curves are smoother in

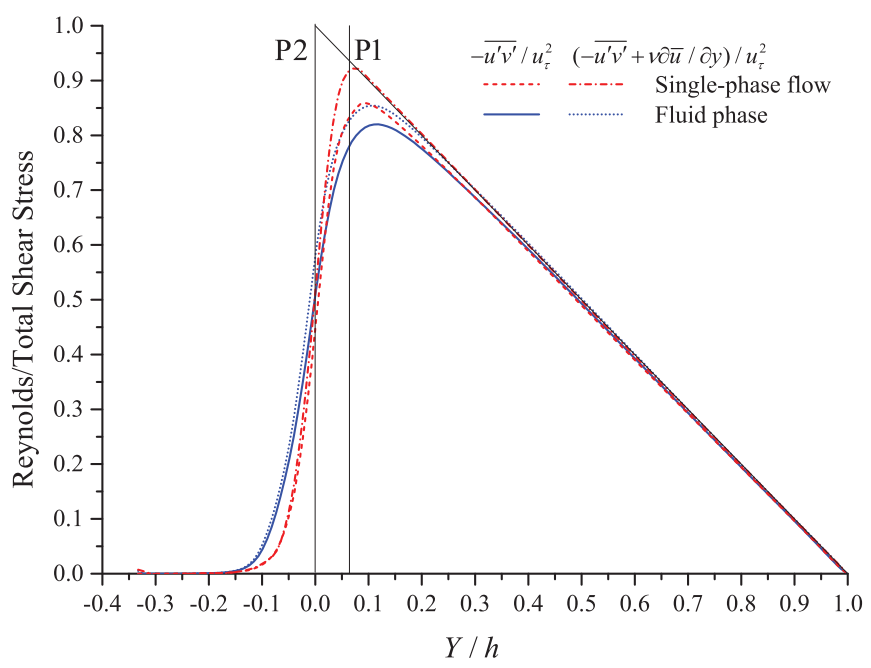

FIG. 8. (Color online) Reynolds shear stress $-\overline{u^{\prime} v^{\prime}} / u_{\tau}^{2}$ and total shear stress $\left(-\overline{u^{\prime} v^{\prime}}+v \partial \bar{u} / \partial y\right) / u_{\tau}^{2}$ of the single-phase and two-phase flow cases. The prime denotes the turbulent fluctuation of a quantity. The inclined solid line represents the profile of total shear stress for fully developed smooth-wall flow. P1 and P2 are two wall-parallel planes and their positions are shown in Fig. 2. 


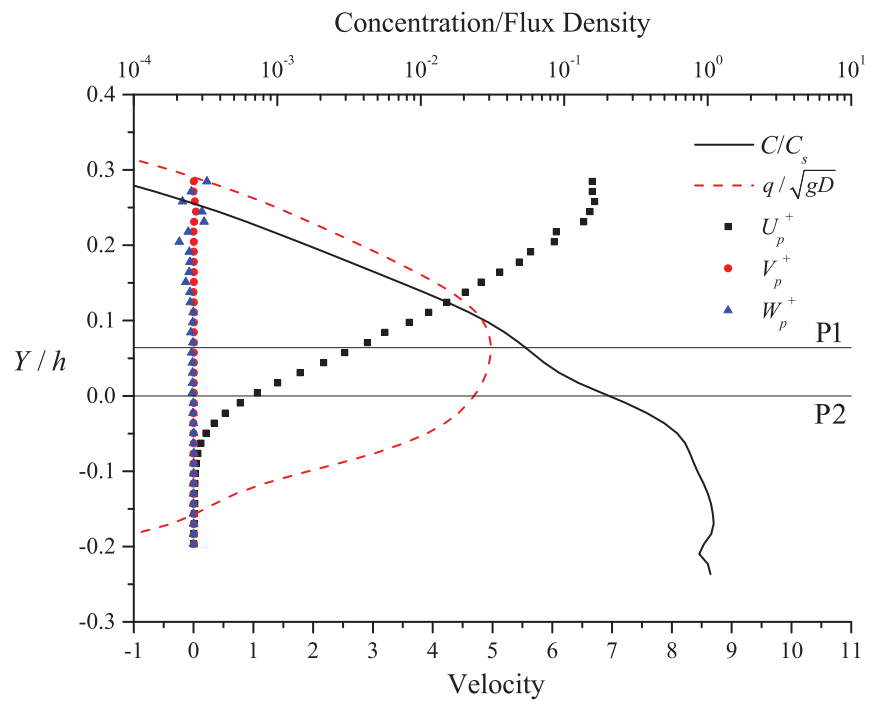

FIG. 9. (Color online) Mean particle velocity profiles, normalized sediment concentration, and normalized volumetric flux density of the dispersed phase. Small particle velocity fluctuations in the top region of the bed-load layer are due to insufficient sampling. P1 and P2 are two wall-parallel planes and their positions are shown in Fig. 2.

the near-wall region compared with those of the single-phase flow which is in agreement with the findings of Kidanemariam et al. [17] although the differences in our results are more pronounced. With the increasing height, the Reynolds and total shear stresses of the fluid phase change from larger to smaller than those of the single-phase flow and the critical point is roughly at P2. The largest reduction of the total shear stress of the fluid phase, compared with that of the single-phase flow, occurs at P1. If we take the total shear stress of the single-phase flow at this position as unity, the percentage of the total shear stress carried by the fluid phase is $90 \%$ and that carried by the dispersed phase is $10 \%$. The shear stress imposed on the dispersed phase is balanced by the friction forces and contact forces between the moving particles. These two values change to $95.6 \%$ and $4.4 \%$ at $Y / h=0.11$, a position where the total shear stress of the fluid phase peaks.

\section{Statistics of translation and rotation of the entrained particles}

Figure 9 shows the binned and time-averaged velocity components of the dispersed phase. The mean vertical and spanwise velocity profiles are almost zero throughout the channel depth, despite the small fluctuations observed in the top region of the bed-load layer. The volumetric flux density function $q$ and the sediment concentration $C$ are also presented in Fig. 9 in which $q$ and $C$ are normalized by $\sqrt{g D}$ and $C_{s}$, respectively, where $C_{s} \approx 0.63$ is the average sediment concentration of the static rough bed. The flux density shows a symmetric profile and peaks at P1. Beyond that, the flux density function and the sediment concentration decrease exponentially with height and agree with the numerical results of Durán et al. [40].

The volumetric sediment flux $q_{s}$ can be calculated as $q_{s}=\int_{0}^{d} q(y) d y$ and the nondimensional volumetric sediment

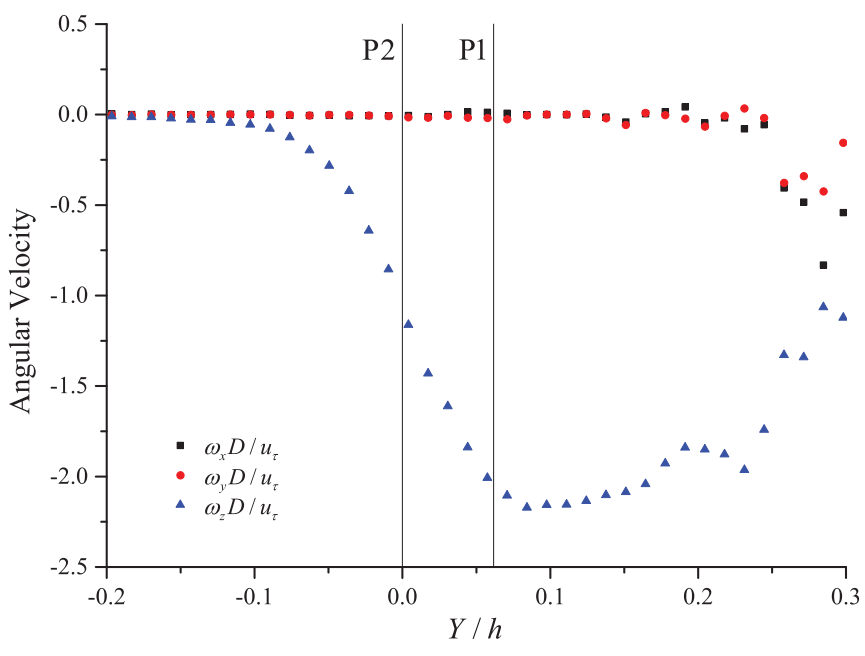

FIG. 10. (Color online) Mean angular velocity (normalized by $u_{\tau} / D$ ) of the dispersed phase. The fluctuations in the top region of the bed-load layer are caused by insufficient sampling. P1 and P2 are two wall-parallel planes and their positions are shown in Fig. 2.

flux is $\phi=q_{s} / \sqrt{\left(\rho_{s} / \rho_{f}-1\right) g D^{3}}=0.0327$, which agrees well with experimental data and several well-known bed-load transport equations' values, ranging from 0.01 to 0.04 , at $\Theta=0.065$ (compiled by Wiberg and Smith [41]; refer to Fig. 7 of their work). However, if $q_{s}$ is calculated according to $q_{s}=$ $\pi D^{3} /(6 A) \sum_{p} \bar{u}_{p}^{+}$, where $A$ is the channel area and $\sum_{p} \bar{u}_{p}^{+}$is the sum of mean particle velocity over all spheres, $\phi$ becomes 0.0335 . It should be noted that $\bar{u}_{p}^{+}$represents the time-averaged streamwise velocity of an individual particle whereas $U_{p}^{+}$ indicates the binned and time-averaged streamwise velocity of the dispersed phase.

Figure 10 shows the binned and time-averaged angular velocities, normalized by $u_{\tau} / D$, of the dispersed phase. The mean streamwise and vertical angular velocities, i.e., $\omega_{x}$ and $\omega_{y}$, are generally zero except fluctuations in the top region of the bed-load layer, while the one in the spanwise direction $\omega_{z}$ decreases with height and reaches a negative plateau with $\omega_{z} D / u_{\tau} \approx 2.2$ before it plummets at $Y / h \approx 0.23$ owing to the insufficient sampling. The negative spanwise angular velocity indicates that the entrained particles predominately rotate clockwise in the $x-y$ plane (flow is from left to right). All standard deviations of the angular velocities show a similar trend except that the spanwise components have a larger value, as shown in Fig. 11.

\section{E. Statistics of particle saltation trajectory}

We investigate further the statistical features of several key parameters of particle saltation trajectory. Each saltation is identified by two successive time instances when the vertical velocity changes from negative to positive. However, to discriminate the particle saltation from particle fluctuation near a balanced position, a criterion was applied that the vertical component of the contact force of the colliding particles at the beginning of a saltation should be larger than 1.5 times its submerged weight. To verify that our selection of the criteria was not arbitrary, different values of the criteria, ranging from 


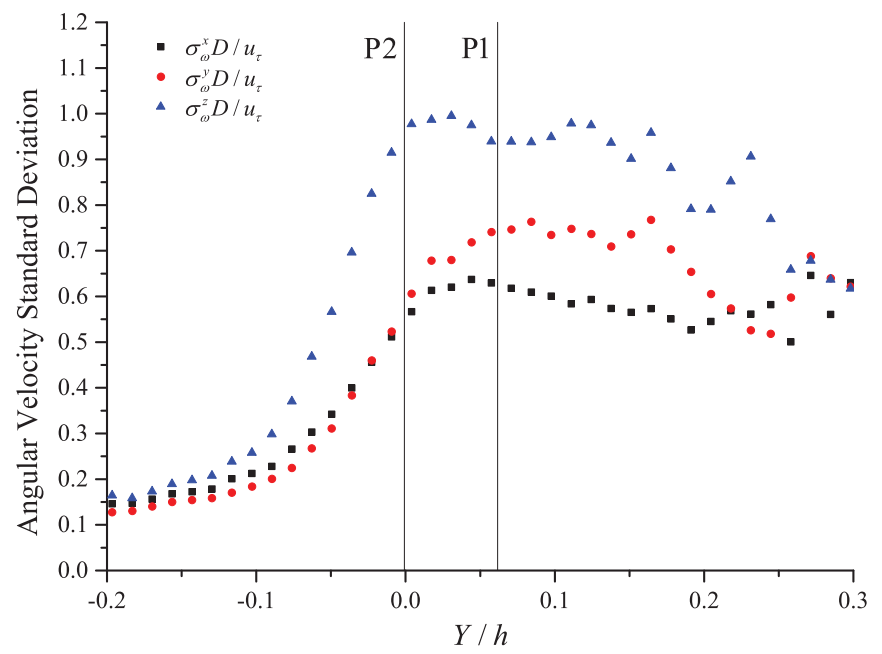

FIG. 11. (Color online) Angular velocity standard deviation (normalized by $u_{\tau} / D$ ) of the dispersed phase. The fluctuations in the top region of the bed-load layer are caused by insufficient sampling. P1 and P2 are two wall-parallel planes and their positions are shown in Fig. 2.

1.2 to 3.0 times of the submerged weight, were investigated and little difference in the statistical features was observed.

Table II shows the comparison between experimental data and our numerical results. The mean nondimensional particle saltation length $\lambda_{s} / D$ and its standard deviation of our results show good agreement with the experimental data of Niño and García [6] (referred to as NG hereafter) and Abbott and Francis [4] (referred to as AF hereafter) with the transport stages $\tau_{w} / \tau_{w c}$ of all three cases slightly above 1.0 , where $\tau_{w c}$ is the critical wall shear stress for sediment entrainment and calculated using the critical Shields function $\Theta_{c}=0.06$. We also compare our results with those from Niño et al. [7] who investigated the saltation of very large particles of gravel in a flume with a relatively high transport stage of 1.33 and good agreement is achieved. It should be noted that the grain sizes of the four cases differ significantly (natural sand particles with $D=0.53 \mathrm{~mm}$ and $\mathrm{Re}_{D}^{+}=10.6$ in $\mathrm{NG}$, ellipsoidal pea gravel with a nominal diameter $D=8.28 \mathrm{~mm}$ and $\operatorname{Re}_{D}^{+}=523$ in AF, natural gravels with $D=31 \mathrm{~mm}$ and $\mathrm{Re}_{D}^{+}=6200$ in Niño et al. [7], and spheres with $D=1.0 \mathrm{~mm}$ and $\operatorname{Re}_{D}^{+}=86.5$ in our case), which means the normalized saltation length is insensitive to the particle size. A similar conclusion can be deduced for the normalized particle saltation height $h_{s} / D$ although our numerical results are a little lower than those of NG and AF. The nondimensional streamwise velocity of particle saltation $u_{s} / u_{\tau}$ shows a clear dependency on grain size. For large particle size, our numerical results agree well with the data of the gravel transport experiments of Niño et al. [7] and the gravel transport experiments with $D=3.3 \mathrm{~mm}$ and $\operatorname{Re}_{D}^{+}=150$ of Luque and van Beek [42] (also the value calculated using the regression equation recommended in their paper). However, the nondimensional streamwise velocity increases with decreasing grain size, e.g., $u_{s} / u_{\tau}=4.26$ for the case with $D=0.9 \mathrm{~mm}$ and $\operatorname{Re}_{D}^{+}=19.9$ of Luque and van Beek [42] and $u_{s} / u_{\tau}=5.95$ for the case with $D=0.53$ $\mathrm{mm}$ and $\operatorname{Re}_{D}^{+}=10.6$ of NG. This could be attributed to the fact that large-size particles need more momentum to reach a high streamwise velocity. The mean nondimensional time span for a particle saltation is $t_{s} u_{\tau} / D \approx 1.8$. The takeoff angle $\theta_{\text {out }}$ and incident angle $\theta_{\text {in }}$ of the particle saltation trajectory (just before and after collisions) of the present results are higher than those of NG and Niño et al. [7] and could be a result of the fact that the very short-time collisions were not always captured by the video cameras in these experiments, which is likely to introduce some errors in $\theta_{\text {out }}$ and $\theta_{\text {in }}$. The mean dynamic friction coefficient $\mu_{d}=(1+R) \tau_{w} \Delta u_{s} u_{s} / \lambda_{s}$ of our numerical results is 0.158 which agrees well with 0.159 of NG. As a whole, the statistics obtained from the DNS data show excellent agreement with the experimental values and give strong evidence of the high accuracy and fidelity of our numerical results.

TABLE II. Comparison of particle saltation statistics between experimental data and numerical results. $D$ is the particle geometry diameter, $\operatorname{Re}_{D}^{+}$the particle Reynolds number, $\Theta_{c}$ the critical Shields function for sediment motion, $s_{s}$ the density ratio of particle and fluid, $\tau_{w}$ the bed shear stress, $\tau_{w c}$ the critical bed shear stress for sediment motion, $\lambda_{s}$ the mean particle saltation length, $h_{s}$ the mean particle saltation height, $u_{s}$ the mean streamwise particle saltation velocity, $\theta_{\text {in }}$ the mean angle of incidence at collision, $\theta_{\text {out }}$ the mean angle of takeoff at collision, and $\mu_{d}$ is the dynamic friction coefficient.

\begin{tabular}{|c|c|c|c|c|c|}
\hline Variables & Niño and García [6] & Abbott and Francis [4] & Luque and Beek [42] & Niño et al. [7] & Present results \\
\hline$D$ & $0.5 \mathrm{~mm}$ & $8.28 \mathrm{~mm}$ & $0.9 \mathrm{~mm}$ and $3.3 \mathrm{~mm}$ & $31 \mathrm{~mm}$ & $1.0 \mathrm{~mm}$ \\
\hline $\operatorname{Re}_{D}^{+}$ & 10.6 & 523 & 19.9 and 150 & 6200 & 86.5 \\
\hline$\Theta_{c}$ & 0.05 & 0.06 & 0.05 and 0.06 & 0.06 & 0.06 \\
\hline$s_{s}$ & 2.65 & 2.57 & 2.64 & 2.65 & 2.65 \\
\hline$\tau_{w} / \tau_{w c}$ & 1.03 & 1.08 & 1.01 and 1.03 & 1.33 & 1.08 \\
\hline$\lambda_{s} / D$ & $6.43 \pm 3.18$ & 5.93 & - & $5.97 \pm 1.93$ & $6.59 \pm 2.71$ \\
\hline$h_{s} / D$ & $1.01 \pm 0.32$ & 1.03 & - & $0.89 \pm 0.41$ & $0.86 \pm 0.24$ \\
\hline$u_{s} / u_{\tau}$ & $5.95 \pm 1.85$ & - & $\begin{array}{c}3.44 \text { (regression equation) } \\
3.67(D=3.3 \mathrm{~mm}) \\
4.26(D=0.9 \mathrm{~mm})\end{array}$ & $3.29 \pm 1.01$ & $3.61 \pm 1.26$ \\
\hline$\theta_{\text {out }}$ & $27.0 \pm 14.5^{\circ}$ & - & - & $32.1 \pm 17.9^{\circ}$ & $40.5 \pm 24.1^{\circ}$ \\
\hline$\theta_{\text {in }}$ & $13.8 \pm 11.4^{\circ}$ & - & - & $19.2 \pm 8.33^{\circ}$ & $25.2 \pm 9.19^{\circ}$ \\
\hline$\mu_{d}$ & $0.159 \pm 0.281$ & - & - & - & $0.158 \pm 0.148$ \\
\hline
\end{tabular}




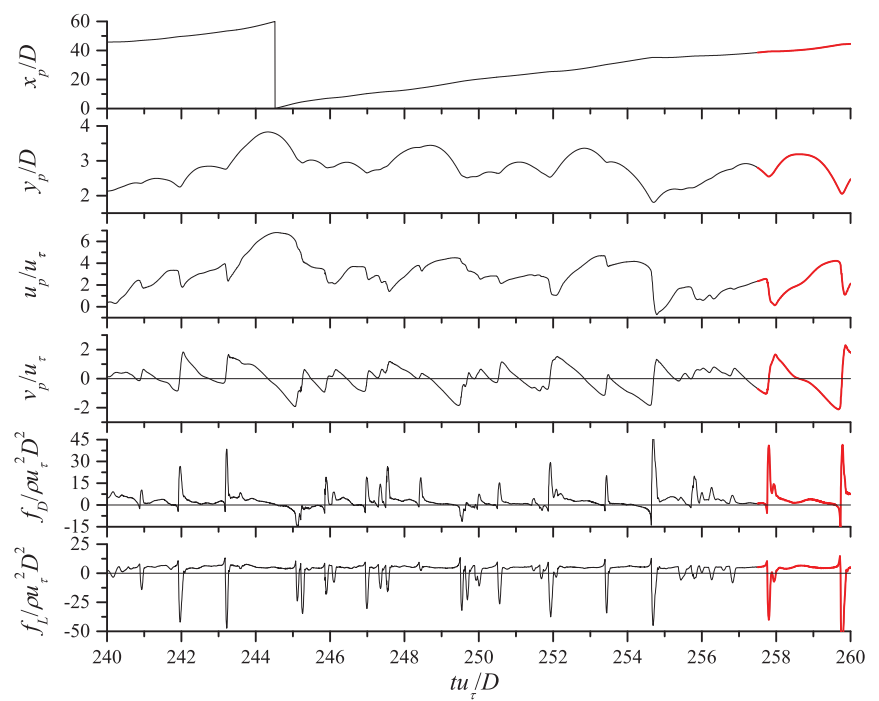

FIG. 12. (Color online) Dynamic responses of a particle during typical continuous particle saltations. $x_{p}$ and $y_{p}$ indicate the instantanous streamwise and vertical coordinates of the particle, respectively; $u_{p}$ and $v_{p}$ represent the instantanous streamwise and vertical velocities of the particle, respectively; and $f_{D}$ and $f_{L}$ are the instantanous drag and lift forces, respectively. Coordinates, velocities, and forces are normalized by particle diatmeter $D$, bed friction velocity $u_{\tau}$, and $\rho u_{\tau}^{2} D^{2}$. Time is normalized by $D / u_{\tau}$. The jump in the streamwise coordinate curve is due to the periodic boundary conditions in which particles moving out of the right boundary of the computational domain were artificially placed at the left boundary. The last saltation is indicated by thicker solid lines in red (dark gray).

\section{DYNAMIC PROCESS AND MECHANISMS OF PARTICLE SALTATION}

Figure 12 shows the time series of a typical continuous saltation of a particle which almost moves through the whole computational domain in the streamwise direction. About ten obvious hops were observed in the nondimensional time span of $t u_{\tau} / D=20$. The vertical velocity decreases linearly between two successive collisions despite small fluctuations caused by the turbulent coherent structures and the interference from other particles passing by. The effects of turbulent coherent structures are less important for the continuous saltation of large particles than that of smaller ones in NG. Large particles obtain vertical momentum mainly from their streamwise momentum at collision, which can be deduced from the strong correlation between the abrupt decrease in the streamwise velocity and the sudden increase in the vertical velocity; turbulent structures only cause small fluctuations in the hydrodynamic force and velocity curves.

To clearly demonstrate the saltation process, a typical saltation $\left(257.5<t u_{\tau} / D<260\right)$, as shown in Fig. 13, has been extracted from Fig. 12. Six critical instances in time, indicated by the vertical lines in Fig. 13, are selected while corresponding particle positions and pressure distribution on particle surface are presented in Fig. 14.

The first instance the authors chose is when the vertical velocity of the particle changes from negative to positive and corresponds to the beginning of a saltation. At this time, the particle reaches the lowest position in a saltation and the

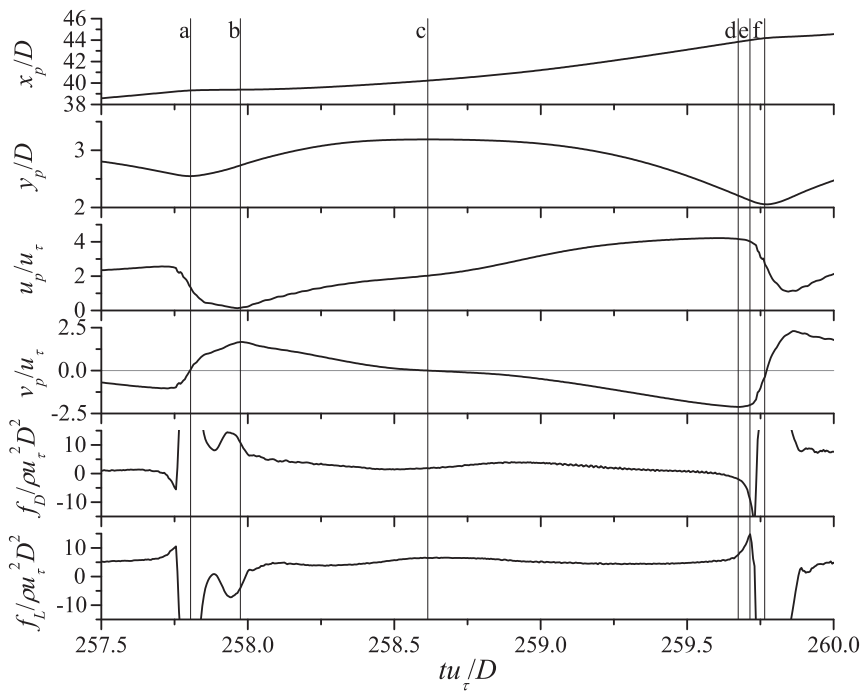

FIG. 13. Particle dynamic responses during a typical saltation. The saltation is selected as the last one in Fig. 12. The vertical lines indicate six critical instances in time.

drag and lift forces reach their peak positively and negatively. Figure 14(a) clearly shows a low-pressure region under the bottom of the particle and a high-pressure region on the top windward side of the particle.

The second moment in time corresponds to the instant when the vertical velocity reaches its maximum and the streamwise velocity reaches its minimum. This could be recognized as the end of a collision and the beginning of free flying of the particle. The velocity difference between the fluid and the rebounding particle is so large that a significant flow separation and a corresponding low pressure at the top lee side of the particle are observed, as shown in Fig. 14(b). The small fluctuations in the drag and lift force curves are caused by the vortex shedding in the wake. However, not all saltations have these force fluctuations which depend upon the particle's rebounding velocity and the surrounding turbulent flow.

Instance 3 is for the free-flying stage during which only the turbulence structures affect the particle saltation. This can be seen in Fig. 14(c) where there is a low-pressure region related to turbulence structures in the outer flow region passing by the moving particle which cause slightly larger lift and smaller drag forces. Apart from the effect of the turbulence fluctuations, the lift force is almost constant in the whole freeflying stage.

Instance 4 [Fig. 14(d)] is characterized by the largest negative vertical velocity and can be considered as the end of free flying and the beginning of the collision. The reason for the decreasing drag and increasing lift force at this instant could be that the landing particles are more likely to hit on the upslope side of the bed particles and the resulting high pressure in the gaps generates a negative drag and a positive lift force.

Instance 5 [Fig. 14(e)] corresponds to the small negative and positive peaks in the drag and lift force curves, respectively. The pressure distribution at this moment is similar to that of instance 4 except that a slightly higher pressure is observed on 

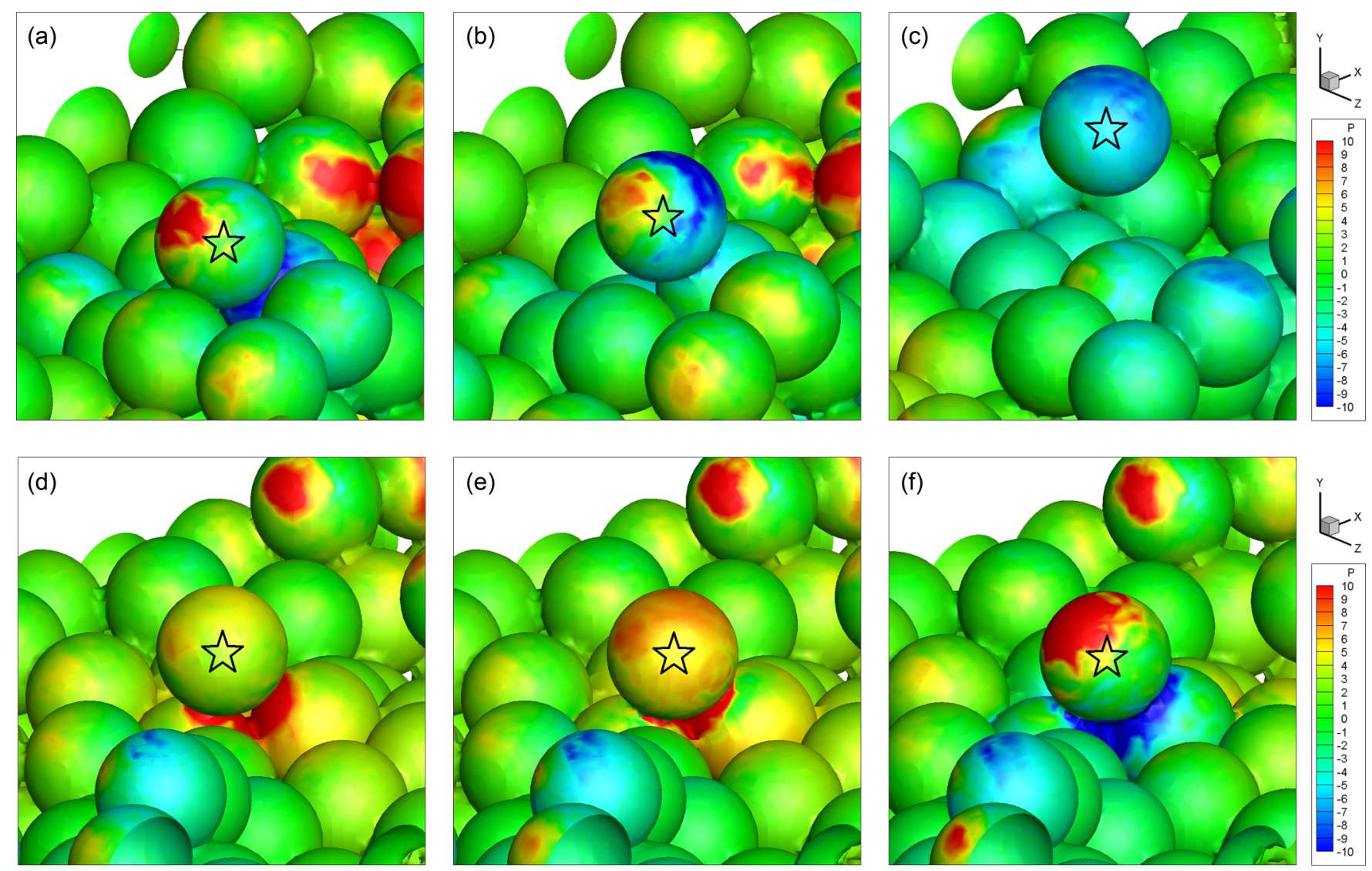

FIG. 14. (Color online) Pressure contours on particle surface during a typical saltation. Time of the sequence corresponds to the six critical instances in Fig. 13 and the target particle is marked with a star.

the top particle surface, due to the decelerating of the particle in the streamwise direction.

Instance 6 [Fig. 14(f)] is the end of the saltation and the pressure distribution is similar to that of instance 1 .

The continuous saltation process observed in this study supports the conclusions of NG in that collision rebounding is possible but argues against the statements by Gordon et al. [43] and AF denying such a possibility. Gordon et al. [43] found in their "one-dimensional" particle saltation experiments that a moving particle generally did not bounce off a stationary one on a rough bed, but rolled over it for some distance. AF stated that clear rebounding, without rolling, is rare, and that most takeoff velocities at the start of a trajectory are uninfluenced by the previous impact. These views are contrary to our findings and the discrepancy could be due to the different particle densities used. In the experiments of $\mathrm{AF}$, various particle-fluid density ratios from 1.2 to 2.6 were used whereas Gordon et al. [43] investigated plastic particles with a density ratio of 1.3 . However, in our study and the study of NG, the density ratio is 2.65 . Smaller densities lead to smaller particle inertias which causes the particles to be less able to overcome the hydrodynamic resistance at collision and thus tend to slide and roll. The side-wall effects of the one-dimensional experiments [43] carried out in a flume with two side walls placed only about one particle diameter from each other for easy observation could be another possible reason for the differences owing to significant fluid damping.
The mechanisms for the entrainment and the subsequent continuous saltation of large-grain-size particles are different. As shown by Dwivedi et al. [44] and our previous work [19], turbulent coherent structures, especially sweep structures, play a significant role in sediment entrainment. Large particles are expelled off the rough bed by the pressure gradient generated by the turbulence structures. However, for the subsequent continuous saltation of large particles, collision parameters, such as incident angle, local rough-bed packing arrangement, particle density, fluid viscosity, etc., are crucial and the turbulent coherent structures are weakened or destroyed by the entrained large particles. The generated contact force can be one order of magnitude larger than the hydrodynamic force fluctuations caused by the turbulence structures as shown in Figs. 12 and 13.

\section{CONCLUSION}

In this study, particle saltation in a turbulent channel flow has been numerically investigated using a methodology which combines the DNS of turbulence flow, the FDEM of particle dynamics, and the IBM for the fluid-solid interaction. Firstand second-order statistics of the particle-laden turbulent flow and particle translation and rotation, together with the sediment concentration and the volumetric flux density profiles, have been presented. It was found that the presence of the dispersed phase significantly modifies the statistical features of the 
turbulent flow in the vicinity of the rough bed. For example, our numerical results have shown a distinct velocity lag between the single- and two-phase mean streamwise flow profiles, and the reason has been attributed to a larger particle size and solid volume fraction instead of smaller particles and volume fraction which produce the streaky patterns reported in some previous experimental and numerical studies. Quasistreamwise-aligned streaky flow structures are not observed in the near-wall region and particles scatter on the rough bed owing again to their large size and solid volume fraction. However, turbulent coherent structures recover in the outer flow region where the rough-bed effects disappear and the entrained particles rarely reach. The particles' mean streamwise velocity shows a quasilinear profile, while their mean angular velocity in the spanwise direction is almost constant. Several key parameters of particle saltation trajectory have been calculated and excellent agreement with experimental data was achieved. Time histories of the hydrodynamic forces acting on a typical saltating particle and the dynamic process of particle collisions were also investigated. The dominating continuous particle saltation shows that the collision-rebounding process is possible and that small particle densities could be a possible reason for the absence of such phenomenon in some previous experimental studies. The strong correlation between the abrupt changes in particle streamwise and vertical velocities indicate that the particle's upward momentum is transferred from the streamwise momentum by particle-bed collisions. This shows a clearly different physics comparing with that for particle entrainment in which particle upward momentum is obtained from turbulent coherent structures.

\section{ACKNOWLEDGMENTS}

This work was supported by a Marie Curie International Incoming Fellowship within the 7th European Community Framework Programme (Grant No. PIIF-GA-2009-236457). The first author acknowledges the financial support of the Science Fund for Creative Research Groups of the National Natural Science Foundation of China (Grant No. 51321065), National Natural Science Foundation of China (Grants No. 50809047, No. 51109157, and No. 51009105), and Natural Science Foundation of Tianjin (Grants No. 12JCQNJC02600, No. 12JCQNJC04900, and No. 12JCQNJC05600). We thank UKTC for HECToR computer time and facilities.
[1] Y. Niño and M. García, Water Resour. Res. 30, 1915 (1994).

[2] R. J. Bialik, V. I. Nikora, P. M. Rowinski, and W. Czernuszenko, in Proceedings of the International Conference on Fluvial Hydraulics, River Flow 2010, Braunschweig, Germany, edited by A. Dittrich, K. Koll, J. Aberle, and H. Geisenhainer (Bundesanstalt für Wasserbau, Karlsruhe, Germany, 2010), pp. 105-112.

[3] L. C. van Rijn, J. Hydraul. Div., Am. Soc. Civ. Eng. 110, 1431 (1984).

[4] J. E. Abbott and J. R. D. Francis, Philos. Trans. R. Soc., A 284, 225 (1977)

[5] Y. Niño and M. H. García, J. Fluid Mech. 326, 285 (1996).

[6] Y. Niño and M. H. García, J. Hydraul. Div., Am. Soc. Civ. Eng. 124, 1014 (1998).

[7] Y. Niño, M. García, and L. Ayala, Water Resour. Res. 30, 1907 (1994).

[8] H. Y. Lee and I. S. Hsu, J. Hydraul. Div., Am. Soc. Civ. Eng. 120, 831 (1994).

[9] H. Y. Lee, Y. H. Chen, J. Y. You, and Y. T. Lin, J. Hydraul. Div., Am. Soc. Civ. Eng. 126, 691 (2000).

[10] H. Y. Lee, J. Y. You, and Y. T. Lin, J. Hydraul. Div., Am. Soc. Civ. Eng. 128, 443 (2002).

[11] C. Ancey, F. Bigillon, P. Frey, J. Lanier, and R. Ducret, Phys. Rev. E 66, 036306 (2002).

[12] C. Ancey, T. Böhm, M. Jodeau, and P. Frey, Phys. Rev. E 74, 011302 (2006).

[13] F. Osanloo, M. R. Kolahchi, S. McNamara, and H. J. Herrmann, Phys. Rev. E 78, 011301 (2008).

[14] Y. Niño and M. García, Hydrol. Processes 12, 1197 (1998).

[15] C. Chan-Braun, M. García-Villalba, and M. Uhlmann, J. Fluid Mech. 684, 441 (2011).

[16] C. Chan-Braun, M. García-Villalba, and M. Uhlmann, High Perform. Comput. Sci. Eng. 10, 295 (2011).
[17] A. G. Kidanemariam, C. Chan-Braun, T. Doychev, and M. Uhlmann, New J. Phys. 15, 025031 (2013).

[18] X. Shao, T. Wu, and Z. Yu, J. Fluid Mech. 693, 319 (2012).

[19] C. Ji, A. Munjiza, E. Avital, J. Ma, and J. J. R. Williams, Phys. Fluids 25, 056601 (2013).

[20] T. G. Thomas and J. J. R. Williams, J. Wind Eng. Ind. Aerodyn. 67-68, 155 (1997).

[21] K. M. Singh, N. D. Sandham, and J. J. R. Williams, J. Hydraul. Div., Am. Soc. Chem. Eng. 133, 386 (2007).

[22] J. Ma and J. J. R. Williams, J. Hydro-environ. Res. 3, 2 (2009).

[23] C. S. Peskin, J. Comput. Phys. 10, 252 (1972).

[24] C. Ji, A. Munjiza, and J. J. R. Williams, J. Comput. Phys. 231, 1797 (2012).

[25] A. Munjiza, D. R. J. Owen, and N. Bicanic, Eng. Comput. 12, 145 (1995).

[26] A. Munjiza and K. R. F. Andrews, Int. J. Numer. Methods Eng. 49, 1377 (2000).

[27] J. Xiang, A. Munjiza, J. P. Latham, and R. Guises, Eng. Comput. 26, 673 (2009).

[28] A. M. Ardekani and R. H. Rangel, J. Fluid Mech. 596, 437 (2008).

[29] A. Munjiza, The Combined Finite-discrete Element Method (John Wiley \& Sons, Chichester, UK, 2004).

[30] A. Munjiza, E. Knight, and E. Rougier, Computational Mechanics of Discontinua (Wiley, Chichester, UK, 2011).

[31] B. Hofland, Ph.D. thesis, Delft University of Technology, Netherlands, 2005.

[32] J. A. Simeonov and J. Calantoni, Int. J. Multiphase Flow 46, 38 (2012).

[33] D. Kaftori, G. Hetsroni, and S. Banerjee, Phys. Fluids 7, 1107 (1995). 
[34] A. Taniere, B. Oesterle, and J. C. Monnier, Exp. Fluids 23, 463 (1997).

[35] K. T. Kiger and C. Pan, J. Turbul. 3, N19 (2002).

[36] M. Righetti and G. P. Romano, J. Fluid Mech. 505, 93 (2004)

[37] M. Muste, K. Yu, I. Fujita, and R. Ettema, Environ. Fluid Mech. 9, 161 (2009).

[38] J. Jiménez, Annu. Rev. Fluid Mech. 36, 173 (2004).

[39] G. Hetsroni and R. Rozenblit, Int. J. Multiphase Flow 20, 671 (1994).
[40] O. Durán, B. Andreotti, and P. Claudin, Phys. Fluids 24, 103306 (2012).

[41] P. L. Wiberg and J. D. Smith, J. Hydraul. Eng. 115, 101 (1989).

[42] R. F. Luque and R. van Beek, J. Hydrol. Res. 14, 127 (1976).

[43] R. Gordon, J. B. Carmichael, and F. J. Isackson, Water Resour. Res. 8, 444 (1972).

[44] A. Dwivedi, B. W. Melville, A. Y. Shamseldin, and T. K. Guha, Water Resour. Res. 47, W01509 (2011). 